\title{
Lattice Instability in High Temperature Superconducting Cuprates and FeAs Systems: Polarons Probed by EXAFS
}

\author{
H. Oyanagi ${ }^{1}$ and C. J. Zhang',2 \\ ${ }^{1}$ Photonics Research Institute, National Institute of Advanced Industrial Science and Technology, 1-1-1 Umezono, \\ Tsukuba, Ibaraki 305-8568, Japan \\ ${ }^{2}$ High Magnetic Field Laboratory, Hefei Institute of Physical Sciences, Chinese Academy of Sciences, P.O. Box 1110, \\ 350\# Shushan Road, Hefei 230031, China \\ Correspondence should be addressed to H. Oyanagi, h.oyanagi@aist.go.jp and C. J. Zhang, zhangcj@hmfl.ac.cn
}

Received 11 June 2009; Accepted 7 October 2009

Academic Editor: Dragan Mihailovic

Copyright (๑) 2010 H. Oyanagi and C. J. Zhang. This is an open access article distributed under the Creative Commons Attribution License, which permits unrestricted use, distribution, and reproduction in any medium, provided the original work is properly cited.

Carrier-induced lattice distortion (signature of polaron) in oxypnictide superconductors is found by an instantaneous local probe, extended X-ray absorption fine structure (EXAFS). Polaron formation is detected as two distinct nearest neighbor distances (FeAs), implying an incoherent local mode that develops coherence at the critical temperature. Comparing the results with the unusual lattice response in cuprate superconductors, intimate correlation between evolution of local lattice mode and superconductivity is revealed. The results suggest that strong electron-lattice interaction is present as a common ingredient in the microscopic mechanism of superconducting transition. The effect of magnetic impurity atoms in cuprates further indicates that magnetic scattering becomes diluted as long as polaron formation is conserved. We argue that polaron coherence dominates electrical conduction and magnetic interaction in oxypnictide and cuprate superconductors.

\section{Introduction}

While the microscopic mechanism of high temperature superconductivity (HTSC) is still in mystery more than 23 years after the discovery [1], recently reported superconductivity in fluorine-doped $\mathrm{LaFeAsO}$ (LFAO) [2] has revived interests in the research of HTSC. Replacing oxygen by fluorine or oxygen vacancy control introduces charge (electrons) carriers which are transferred from the La-O(F) "charge reservoir" layer to the Fe-As conducting layer as illustrated in Figure 1. Superconductivity emerges as the F-doping concentration exceeds about 5\%, away from nondoped antiferromagnetic (AFM) parent phase. In spite of similarity in the phase diagram with that of cuprates, that is, superconducting phase near the AFM phase, the mechanism of superconductivity is still unclear, allowing diversity and contradictions in theoretical models. A conventional phonon mechanism is unlikely as the DFT calculations indicated a weak electron-phonon coupling; yet, the purely electronic mechanism [3] is also less likely than the case of cuprates [4] as the Hubbard $U$ is not large $(U \sim 5 \mathrm{eV})$ [5]. Recent muon spin rotation experiments suggest possibility of multigap BCS-type superconductivity in $\mathrm{Ba}_{0.6} \mathrm{~K}_{0.4} \mathrm{Fe}_{2} \mathrm{As}_{2}$ [6].

A polaron is formed when an electron is strongly coupled to the atoms in a crystal. Reviewing article on a polaronic mechanism of superconductivity is elsewhere [7]. In manganites, coherent polaron condensation is believed to be the driving mechanism of colossal magnetoresistance (CMR) phenomena [8]. Strong coupling between electrons and lattice in HTSC cuprates is demonstrated by angleresolved photoemission spectroscopy (ARPES) [9]. Reflecting these backgrounds, renewed interests on the role of polaron in HTSC mechanism are accumulating. Here we describe lattice effects in oxypnictide [10] studied by means of a local probe, extended X-ray absorption fine structure (EXAFS) and compared with the results for a typical cuprate $\mathrm{La}_{2-x} \mathrm{Sr}_{x} \mathrm{CuO}_{4}$ (LSCO) [11]. Previously, unusual carrierinduced local displacement found for LSCO was interpreted as a nanometer-scale self-organization (stripe) $[12,13]$. In contrast, we take lattice instability as polarons as predicted by theoretical models [14-17]. As the magnitude of EXAFS oscillations is in the order of $10^{-2}$, a highly efficient 


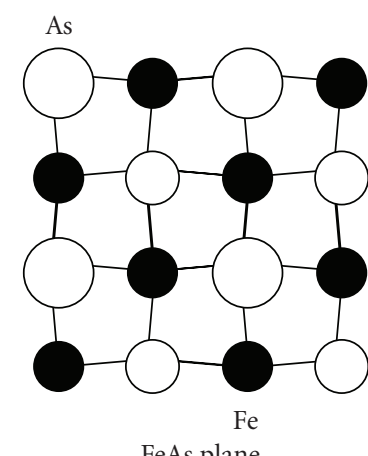

FeAs plane

(a)

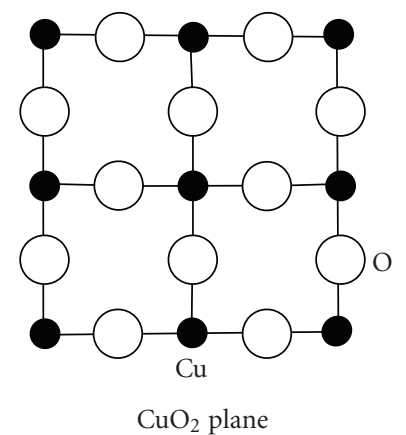

(b)
Figure 1: In-plane structure of the conducting layer in $\mathrm{LaFeAsO}_{1-x} \mathrm{~F}_{x}$ (a) and $\mathrm{La}_{1-x} \mathrm{Sr}_{x} \mathrm{CuO}_{4}$ (b). Use of polarization dependence $(E / / a b)$ XAS measurement can probe the planar local lattice projected onto the $a b$-plane.

segmented detection is needed [18]. We have developed a state-of-the-art pixel X-ray detector to obtain high-quality EXAFS data for single crystals [19]. As chemical doping (substitution with heterovalent atom) modifies lattice itself, and ambiguity is always present, we took special approaches to control the critical temperature without influencing lattice, that is, (i) uniaxial strain dependence [20-22] and (ii) magnetic impurity effect [23].

\section{Experimental}

EXAFS experiments applied to bulk single crystals where the two polarization geometries $(E / / a b, E / / c)$ in principle provide in-plane and out-of-plane information on the radial distribution function (RDF) of the $\mathrm{CuO}_{2}$ plane, respectively. Principle of EXAFS is simple but extreme care should be taken when applied to single crystalline samples. Difficulty in polarized experiments on as-grown single crystals is overcome by a fluorescence detection method that measures an emitted photon flux rather than a transmitted beam intensity. In monitoring fluorescence yield spectra for single crystals, strong diffractions often prevent reliable data taking. Experimental difficulty becomes intensified for thin film single crystals because of strong interference of substrate. Here we use segmented fluorescence monitoring to discriminate signal from noise. A novel germanium 100-pixel array detector (PAD) developed for this purpose is used, collecting signals over a segmented solid angle [24]. All EXAFS data were recorded for bulk, 100-nmthick film LSCO, and polycrystalline $\operatorname{LFAO}(\mathrm{F})$ samples at the Photon Factory. The energy and maximum electron current of storage ring were $2.5 \mathrm{GeV}$ and $400-500 \mathrm{~mA}$, respectively. A directly water-cooled silicon (111) doublecrystal monochromator was used, covering the energy range 4-25 keV. The energy resolution was better than $2 \mathrm{eV}$ at $9 \mathrm{keV}$, calibrated from the near-edge features of copper metal at the Fermi energy, $E_{f}(8.9803 \mathrm{keV})$. Single crystal sample was attached to an aluminum holder with a strain-free glue and cooled down using a closed-cycle He refrigerator (cooling power $2 \mathrm{~W}$ at $20 \mathrm{~K}$, stability $\pm 0.1 \mathrm{~K}$ ) on a highprecision goniometer (Huber 420). As a typical magnitude of normalized EXAFS oscillations is several \%, each data set must have photon statistics better than $0.1 \%$ or $10^{6}$ photons. In a typical experiment, each data point collects average $3.6 \times 10^{7}$ photons making statistical (nonsystematic) noise negligibly small. On the other hand, the most serious noise is systematic origin arising diffractions which distort a fluorescence detector, in particular highly directional Laue diffractions for a single crystalline sample. The advantage of segmented fluorescence detection is that fluorescence signal purity can be monitored over a wide solid angle. Use of segmented detector minimizes both systematic and unsystematic noise leading to a dramatically reduced magnitude of error bar. Repeated scans (about six runs) also reduce systematic error arising from incident beam instability intrinsic to insertion devices. For reliable data taking, non-statistic (systematic) error should be minimized for which segmented X-ray detection and repeated scans are useful. Fluorescence yield spectra for all channels of PAD were monitored in real time and the effect of scattering was inspected. By choosing a proper incidence angle (1 deg) corresponding to a film thickness and further adjustment of orientation, the substrate scattering effect is completely removed. A typical LSCO bulk single crystal grown by TSFZ method was $2 \mathrm{~mm} \times 2 \mathrm{~mm} \times 1 \mathrm{~mm}$ in dimension [11]. Thin film single crystals $(10 \mathrm{~mm} \times 10 \mathrm{~mm} \times 100 \mathrm{~nm})$ were grown on single crystal substrates by molecular beam epitaxy (MBE) [19]. Using a closed cycle He refrigerator, sample temperature is controlled to a temperature error within $1 \mathrm{~K}$. Polycrystalline samples $\mathrm{LaFeAsO}_{1-x} \mathrm{~F}_{x}(x=0,0.07)$ were prepared by solid-state synthesis as described elsewhere [2] and unpolarized EXAFS data were recorded at various temperatures [10].

\section{Results and Discussion}

3.1. LaFeAsO System. Parent LFAO has a double layer structure where the conducting FeAs layer is adjacent to the reservoir LaO layer in close similarity with LSCO where more two-dimensional $\mathrm{CuO}_{2}$ and $\mathrm{LaO}$ planes play their roles $[1,2]$. Figure 1 illustrates the $a b$-plane view of the crystal structures for the FeAs layer in LFAO and the $\mathrm{CuO}_{2}$ plane in LSCO. In the FeAs layer, Fe and As atoms have tetrahedrally coordinated each other resulting in a more three-dimensional network while in LSCO copper atoms are fourfold coordinated by oxygen atoms within the plane and two apical oxygen atoms along the $c$-axis. EXAFS is an "incoherent" In HTSC research; local probes are often categorized into (i) static incoherent (NMR), (ii) dynamical coherent (X-ray and neutron scattering), and (iii) dynamical incoherent (XAS, Raman scattering) techniques. Here "incoherent" is local probe means it observes spatiallyincoherent displacement which is not detected by a conventional diffraction techniques. In contrast, the disadvantage of XAS is that both static and dynamical displacements are observed and cannot be distinguished. local probe that arises from interference of incoming (scattered) and outgoing photoelectrons at the excited atom; local distortions of near 


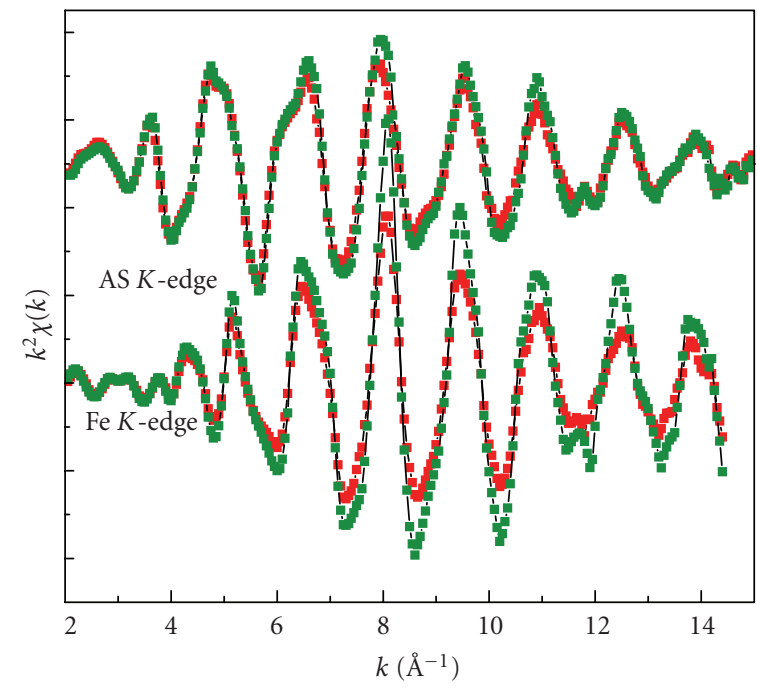

(a)

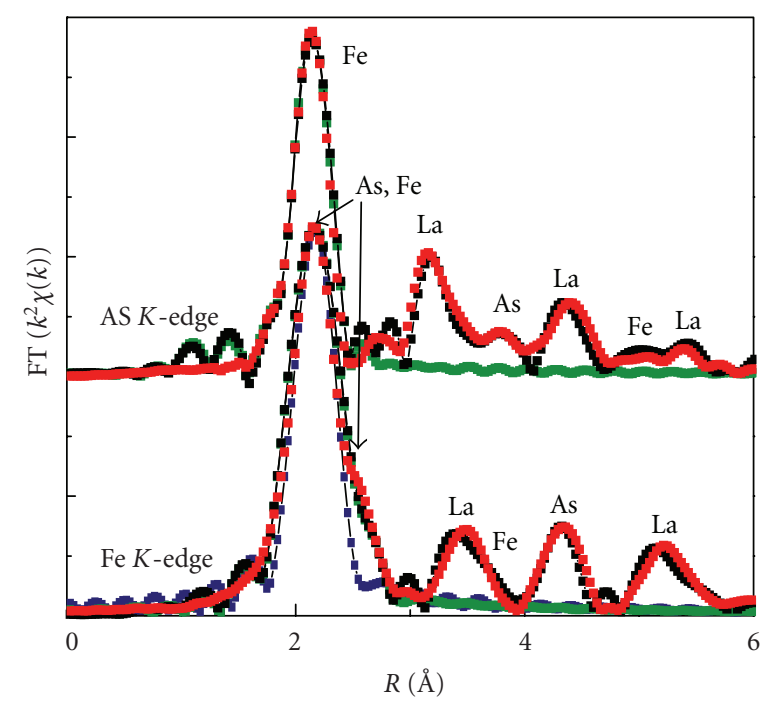

(b)

Figure 2: (a) The Fe $K$ - and As $K$-edge EXAFS oscillations for $\mathrm{LaFeAsO}_{1-x} \mathrm{~F}_{x}(x=0.07)$ plotted as a function of photoelectron wave number $k$. Red and green marks indicate the data measured at $300 \mathrm{~K}$ and $10 \mathrm{~K}$, respectively, with a fluorescence detection mode. (b) Fourier transform magnitude functions for the same data. Red marks indicate the simulated (fitted) curve and black marks represent the experimental data. Green marks denote the experimental nearest neighbour contribution while blue marks represent theoretical calculation for the nearest neighbour Fe-As correlations, taken from [10].

neighbor atoms with static and dynamic nature are sensitively detected as an interference (beat) of $k$-dependent oscillations $\left(k<15 \AA^{-1}\right)$, where $k$ is a wave vector of photoelectron. Complex Fourier transform (FT) of EXAFS oscillations relates to the radial distribution function (RDF) if phaseshift of photoelectron scattering is corrected. Experimental EXAFS oscillations around $\mathrm{Fe}$ and As atoms are compared to those simulated by FEFF7 [25] based on the structural parameters determined by Rietveld analysis and all possible scattering paths including single-scattering and multiplescattering paths [10].

Figures 2(a) and 2(b) illustrate the Fe K- and As KEXAFS oscillations and FT magnitude functions for superconducting $\mathrm{LaFeAsO}_{1-x} \mathrm{~F}_{x}(x=0.07)$, respectively. Curve fitted theoretical curve (red) agreed well with those of experimental data (black). Contributions of the first nearest neighbor shell (green) are back Fourier transformed into $k$ space and analyzed, providing the Fe-As distance and mean square relative displacement (MSRD) $\sigma_{\mathrm{Fe}-\mathrm{As}}^{2}$, denoted here simply as a displacement parameter (DP). The As K-edge FT main peak consists of only the nearest neighbour Fe-As correlation but the Fe K-edge FT has a weak Fe-Fe correlation as a shoulder. We note that the As K-edge data is thus more straightforward in data analysis that directly yields the Fe-As bond distance. In this work, we derived $\sigma_{\mathrm{Fe}-\mathrm{As}}^{2}$ independently from the Fe $\mathrm{K}$-and As K-edge data and confirmed that they agree and show the same temperature anomalies. DP values for the $\mathrm{Fe}-\mathrm{As}$ and $\mathrm{Fe}-\mathrm{Fe}$ correlations are plotted in Figure 3 as a function of temperature where red and green marks represent the Fe-As (Fe K-edge data) and As-Fe (As K-edge data) correlations, respectively. One can notice that undoped sample data (red and green circles) show an indication of magnetic phase transition but lower temperature data are smooth and have no lattice anomalies, in sharp contrast with doped specimen (red and green circles) that shows a complex temperature dependence at low temperatures. In the inset, the Fe-As DP $\left(\sigma_{\mathrm{Fe}-\mathrm{As}}^{2}\right)$ in doped LFAO is compared with that of in-plane $\sigma_{\mathrm{Cu}-\mathrm{O}}^{2}$ in LSCO as a function of normalized temperature. Carrier doping creates a remarkable effect on the Fe-As DP, that is, the Fe-As DP in undoped sample shows no anomaly below the spin-density-wave (SDW) transition temperature $[26,27]$, whereas lattice instability (unusual DP variation with temperature) newly arises as magnetic instability is suppressed in the F-doped sample. Upturn trend beginning at $1.5 T_{c}$ Pseudogap opening and local lattice distortion (polaron formation) are correlated. For optimally doped LSCO, pseudogap opening temperature $\left(T_{\mathrm{po}} \sim 1.5 T_{c}\right)$ which explains why the onset of DP anomaly starts at $1.5 T_{c}$. More recently, in [28], Lee et al. demonstrated that tunneling spectroscopic signature of $d$-wave superconductivity (particle-hole symmetric "octet" of dispersive Bogoliubov QPI modulations) survives up to at least $T \sim 1.5 T_{c}$. and a sharp drop of DP at $T_{c}$ is found in superconducting samples, suggesting strong coupling between the local displacement and superconductivity.

Here lattice instability is defined as unusual behavior of DP, a deviation from a smooth noncorrelated Debyelike function which is observed as carriers are doped. The increase of DP is usually taken as an increase of disorder but here it describes the interference (beat) of bonds having different (short and long) bond lengths. Elongation of bonds is an outcome of local distortion (polaron formation). The inset of Figure 3 shows that a sharp drop of DP occurs at $T_{c}^{\text {onset }}$ in LFAO(F) and LSCO systems, indicating that local 


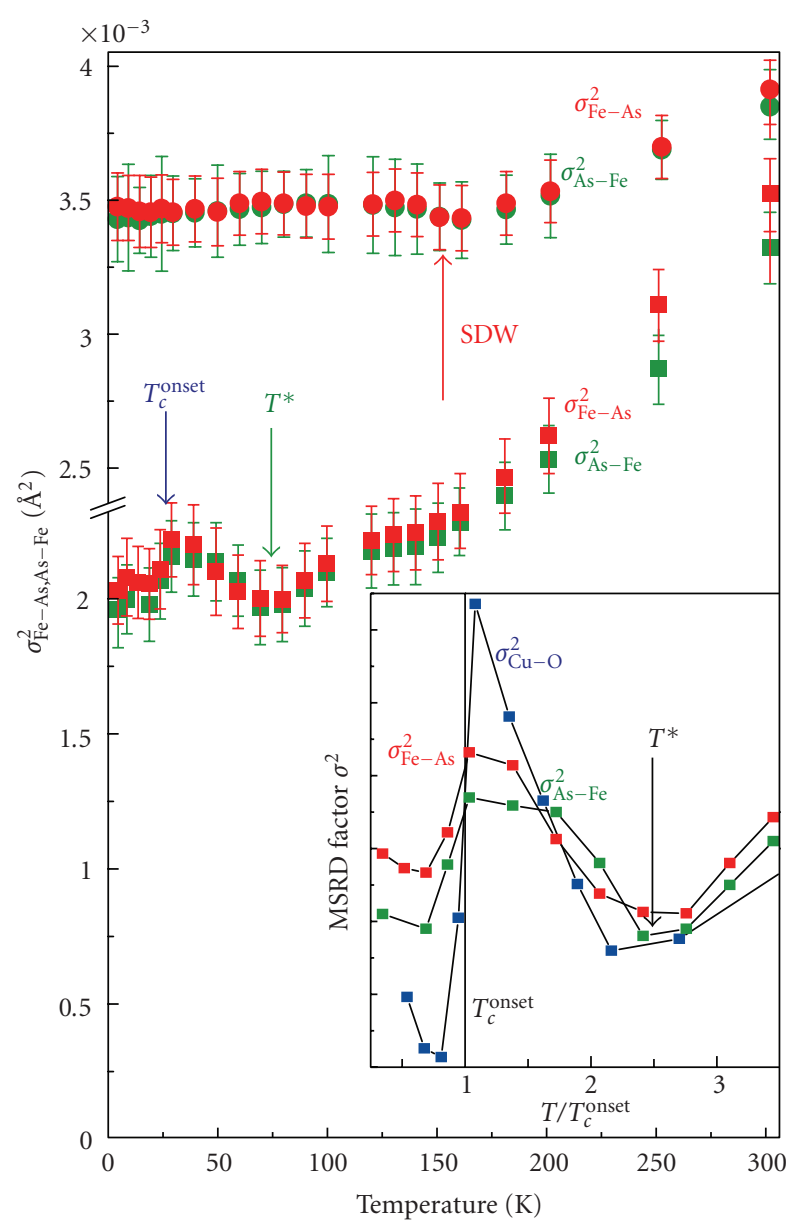

Figure 3: Temperature dependence of the $\mathrm{Fe}-\mathrm{As}$ bond mean-square relative displacements for $\mathrm{LaFeAsO}_{0.93} \mathrm{~F}_{0.07}$ (squares) and $\mathrm{LaFeAsO}$ (circles). The red and green symbols denote the results for the Fe $K$ edge and the As $K$ edge EXAFS data respectively, taken from [10]. The inset shows an enlarged view of low temperature $(T<100 \mathrm{~K})$ mean-square relative displacements (MSRD) for the $\mathrm{LaFeAsO}_{0.93} \mathrm{~F}_{0.07}$ sample plotted as the function of normalized temperature $\left(T / T_{c}^{\text {onset }}\right)$ compared to the result for $\mathrm{La}_{1.85} \mathrm{Sr}_{0.15} \mathrm{CuO}_{4}$.

lattice response directly relates to the microscopic mechanism of superconductivity (development of coherence) while the onset of polaron formation coincides with $T^{*}=70-80 \mathrm{~K}$.

3.2. LaSrCuO System. LSCO is a typical HTSC cuprate with a $\mathrm{K}_{2} \mathrm{NiF}_{4}$-type structure, where copper atoms are coordinated by four in-plane oxygen atoms $\left(\mathrm{O}_{\mathrm{p}}\right)$ and two apical oxygen atoms $\left(\mathrm{O}_{\mathrm{ap}}\right)$. The $\mathrm{CuO}_{6}$ octahedron is elongated along the $c$ axis with two long $(2.40 \AA)$ and four short $(1.89 \AA)$ bonds as a result of Jahn-Teller (JT) distortion [29]. Figure 4 illustrates temperature dependence of the in-plane and out-of-plane $\mathrm{Cu}-\mathrm{O}$ DP $\left(\sigma_{\mathrm{Cu}-\mathrm{O}}^{2}\right)$ for $\mathrm{LSCO}(x=0.15)$ single crystals grown by TSFZ technique. In order to avoid the effect of disorder arising from doping, nonsuperconducting specimen was prepared by substituting copper atom with $5 \% \mathrm{Ni}[11]$. Complex FT and curve fitting were performed for all EXAFS data taken over a wide range in temperature down to $5 \mathrm{~K}$. As temperature is decreased, the superconducting sample

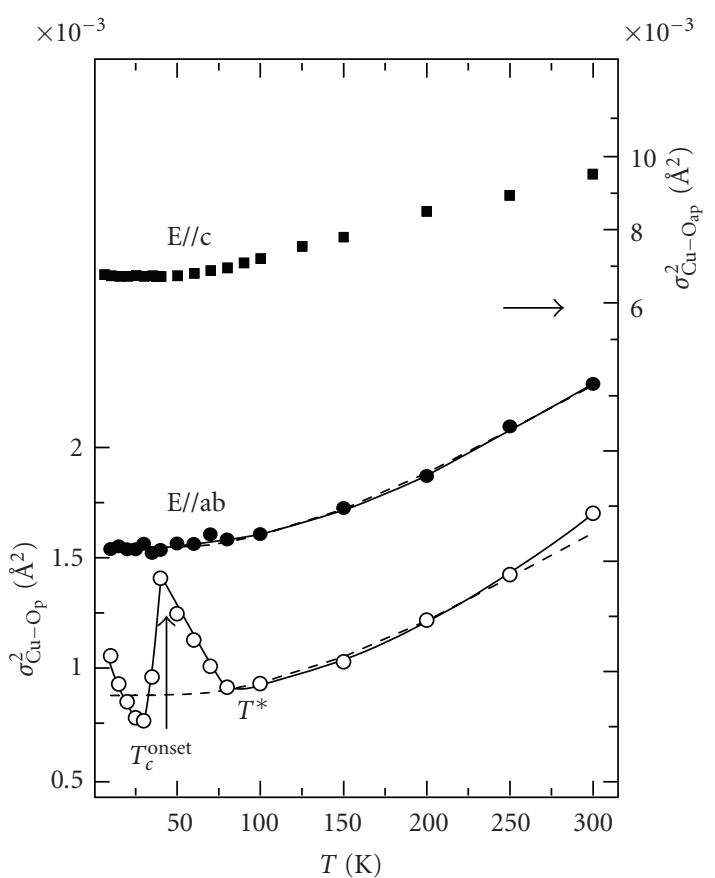

FIgURE 4: Mean-square relative displacement of the $\mathrm{Cu}-\mathrm{O}$ bond in superconducting $\mathrm{La}_{1-x} \mathrm{Sr}_{x} \mathrm{CuO}_{4}(x=0.15)$ with the inplane $(E / / a b)$ and out-of-plane $(E / / c)$ geometries as a function of temperature. Closed and open circles denote the results for $\mathrm{Cu}-\mathrm{O}_{\mathrm{p}}$ (in-plane oxygen) measured for superconducting and nonsuperconducting samples (prepared by doping 3\% Ni), respectively. Closed squares represent the mean-square oxygen displacement for the $\mathrm{Cu}-\mathrm{O}_{\text {ap }}$ (out-of plane oxygen) in superconducting LSCO. Data were taken from [11].

shows lattice instability, that is, an upturn DP beginning at $T^{*}(80 \mathrm{~K})$, which sharply drops at the onset of superconductivity. These features are observed only after carrier doping and are not present in nonsuperconducting sample prepared by magnetic impurity doping [30]. This strict carrier-dependence shows the nature of lattice distortion caused by carrier doping.

Secondly, one may concern whether the apical oxygen atoms behave similarly or not, that is, lattice modes involve apical oxygen atoms or not. Anisotropic nature of lattice instability is studied by comparing the results for the inplane and out-of-plane results obtained by independent polarized experiments. Here we show that the observed polaronic distortion gives rise to two different bond distances distinguished from broadening (disorder). In Figures 5(a) and 5(b), theoretically calculated EXAFS oscillations and FT magnitude functions for undistorted $\mathrm{CuO}_{4}$ and distorted model structures (linked two $\mathrm{CuO}_{4}$ units with $\mathrm{Q}_{2}$ JT or pseudo JT distortions) are illustrated. The appearance of beat feature at $k \sim 12 \AA^{-1}$ corresponds to the bond length difference of $\sim 0.1 \AA^{-1}$ which is observed in the experimental $E / / a b$ $\mathrm{Cu}-\mathrm{O}$ EXAFS oscillations for LSCO shown in Figure 5(c). These results indicate that the in-plane distortion observed as an anomaly in superconducting LSCO is due to the elongated and short bond distances separated by $\sim 0.1 \AA^{-1}$. The outof-plane $\mathrm{Cu}-\mathrm{O}$ bond has no anomaly although the curve 


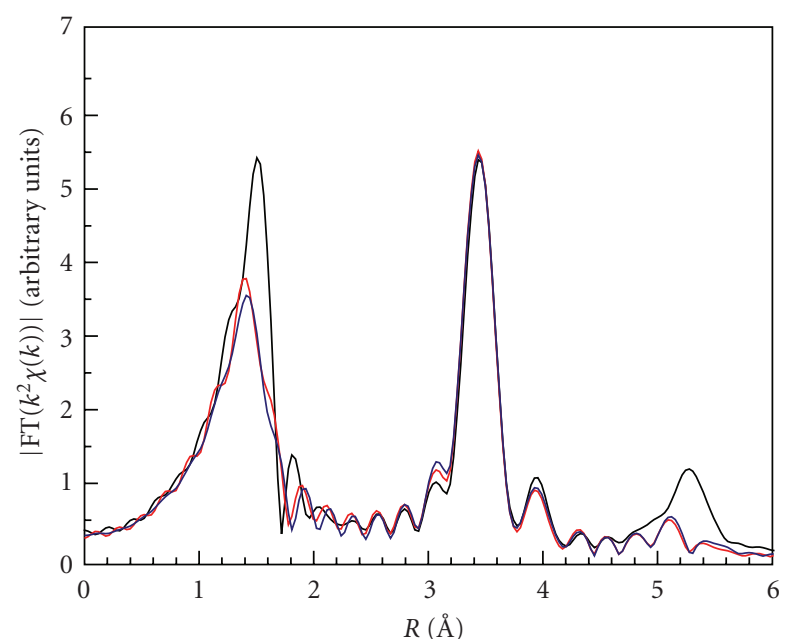

(a)

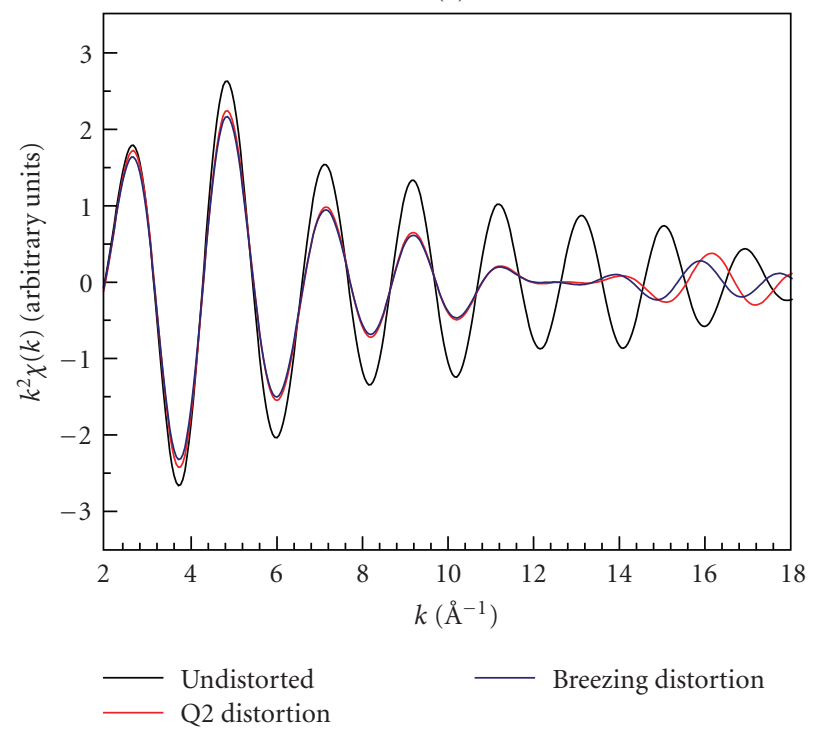

(b)

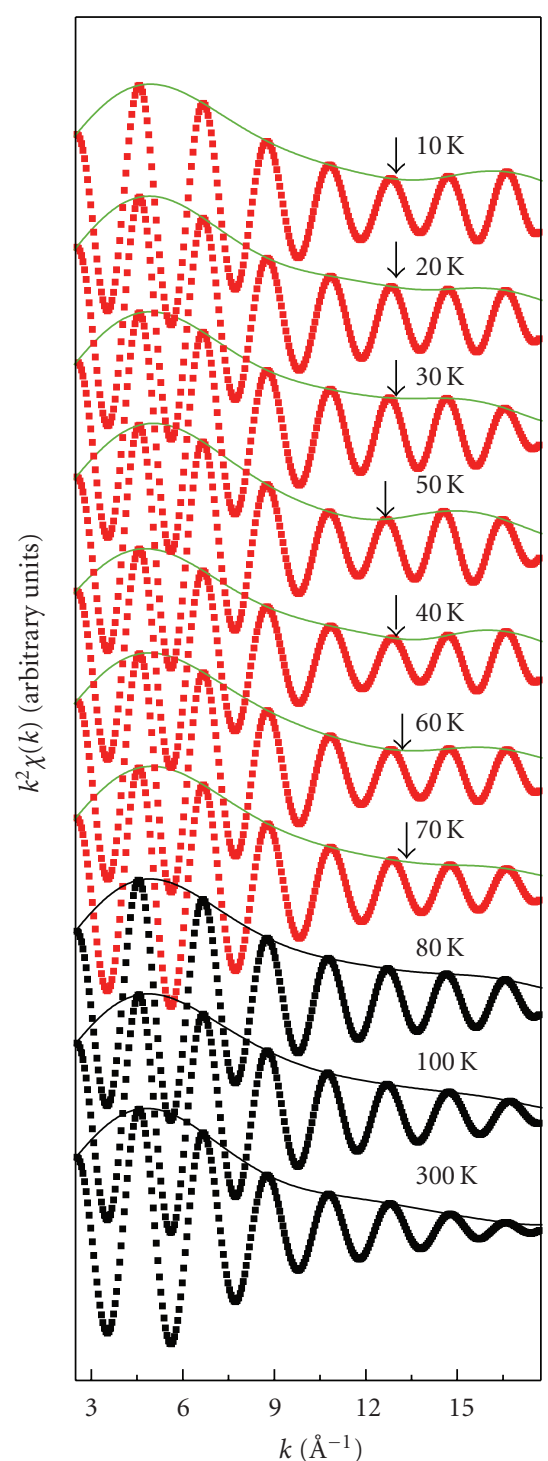

(c)

Figure 5: (a) Fourier transform magnitude curves of simulated E//ab EXAFS oscillations for undistorted (solid line) and distorted CuO ${ }_{4}^{-}$ $\mathrm{CuO}_{4}$ units (red curve: JT Q 2 mode, purple curve: pseudo-JT (breezing) mode distortions). (b) Nearest neighbour contribution of simulated EXAFS oscillations for undistorted and distorted models. Interference of long and short $\mathrm{Cu}-\mathrm{O}$ bonds give rise to beat features at $k \sim 12 \AA^{-1}$. (c) Experimental nearest neighbor Cu-O EXFS curves measured at various temperatures. Beat feature similar to (b) is observed at $k=14 \AA^{-1}$ near $T_{c}(40 \mathrm{~K})$.

fitting analysis for the $c$-axis geometry contains larger error (due to the statistics and multishell fitting procedure) [11], in contrast to the early works which related the anomaly to a low temperature tetragonal (LTT) deformation with correlative displacement of apical oxygen atoms leading to charged stripes.

3.3. Strain Effects in LaSrCuO System. Whether polaron formation probed by the nearest neighbor pair directly relates to superconductivity or not is of particular interest. Hydrostatic high pressure and uniaxial strain experiments are widely used to study the lattice effects on superconductivity. Here we use the mismatch in lattice constants between epitaxial thin film single crystal and substrate that causes compressive or tensile strains. Chemical strain affects the superconducting critical temperature, that is, $T_{c}$ of strained thin film single crystal is modified; LSCO on $\mathrm{LaSrAlO}_{4}$ (LSAO) and $\mathrm{SrTiO}_{3}$ (STO) is $43.4 \mathrm{~K}\left(\Delta T_{c}<1.0 \mathrm{~K}\right)$ and $19.0 \mathrm{~K}\left(\Delta T_{c}<9.0 \mathrm{~K}\right)$, respectively, in contrast to the bulk single crystal value $(35 \mathrm{~K}$ ) [29]. Figure 6(a) shows the $\mathrm{Cu}-\mathrm{O}_{\mathrm{p}}$ DP for LSCO under compressive and tensile strains as a function of temperature. The DP drop clearly shifts with the $T_{c}^{\text {onset }}$ (chemical strain effect) and the magnitude of a sharp DP drop at $T_{c}^{\text {onset }}$ is roughly proportional to the transition temperature, possibly reflecting superfluid density. The correlation of DP drop and the $T_{c}^{\text {onset }}$ is demonstrated in Figure 6(b) plotted against a normalized temperature $\left(T / T_{c}\right)$. 


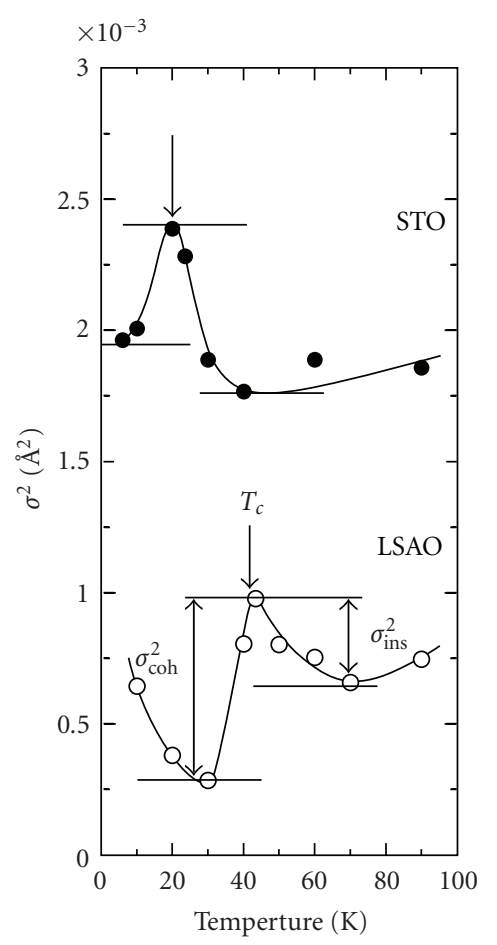

(a)

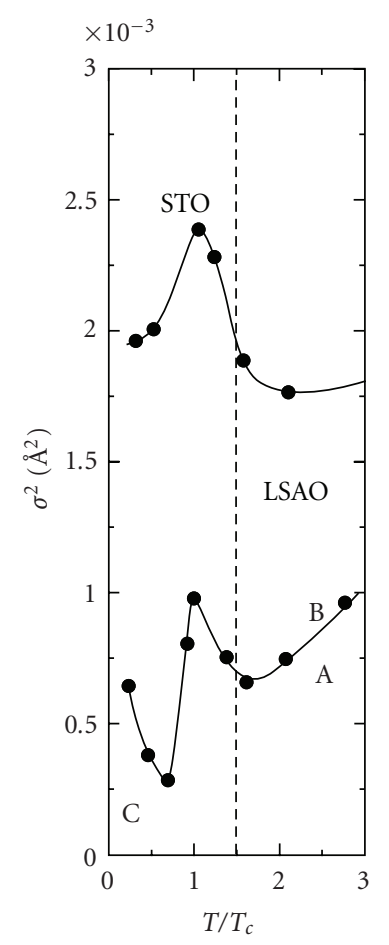

(b)

FIgURE 6: Mean-square relative displacement of the in-plane $\mathrm{Cu}-\mathrm{O}$ bond in thin film single crystal $\mathrm{La}_{1-x} \mathrm{Sr}_{x} \mathrm{CuO}_{4}(x=0.15)$ plotted against temperature (a) and normalized temperature $T / T_{c}(\mathrm{~b})$, taken from [19].

As illustrated in Figures 6(a) and 6(b), the correspondence between the DP maximum and $T_{c}$ is quite good, suggesting that the DP drop is related to superconducting coherence. We take this lattice instability starting at about $1.5 T_{c}\left(T^{*} \sim 1.5 T_{c}\right)$ as a signature of polaron formation, rather than charged magnetic domains with incommensurate spacings (stripes), although nanoscale polarons have strong similarity with stripes in cuprates as the underlying nature has a similar origin (large Hubbard U). Note that the magnitude of DP due to polaron formation becomes enhanced and suppressed under tensile and compressive strain, respectively. This may suggest that the former stabilizes a relevant distortion mode with elongated bonds such as anti JT distortion.

Let us examine possible distortion modes that are consistent with the observed bond splitting. In Figure 7 upper column, possible distortion models with stretched bonds are illustrated. All those models have correlated displacement of apical oxygen atoms and are omitted because of irrelevance of apical oxygens (Figure 4). In contrast, in the lower column, two different types of in-plane distorted pairs, pseudo-JT mode [17] and $\mathrm{Q}_{2}$-type JT mode $[16,31]$, are illustrated. These correlated distortions allow long-range propagation with minimized elastic energy increase. A puzzling question whether the nature of distortion is static or dynamic, that is, leading to localized [17] or extended [16] electron states of polaron, respectively, is unclear as the time scale of EXAFS experiments $\left(10^{-15}\right.$ seconds) is orders of magnitude faster than lattice motions $\left(10^{-12}\right.$ seconds).
Here we discuss implications of lattice fluctuation. It may arise from softening of LO phonons [32-34]. Bondstretching-type LO phonon mode strongly couples with doped holes as a result of $d-p$ mixing or $\mathrm{Cu}-\mathrm{O}$ charge transfer and spin correlation. Femtosecond quasi-particle (QP) lifetime experiments indicated phonon-assisted charge transfer and charge inhomogeneity [35]. Nanometer-scale charge inhomogeneity is one of the fundamental properties of cuprates. Although polarons are expected localized in nature but tunneling [14,35] or percolation [36] may result in extended states with a longer QP lifetime. Here we only note that the two polaron models with different types of in-plane modes (pseudo JT versus $\mathrm{Q}_{2}$-type JT) are consistent with the experimental observation. The former model (pseudo JT distortions with the two $\mathrm{CuO}_{6}$ units) gives $R=1.82 \AA$ and $1.96 \AA$ in good agreement with the EXAFS results [37].

3.4. Magnetic Impurity Effects in LaSrCuO System. Magnetic impurities in cuprate superconductors strongly affect the superconducting properties. We have studied how the $\mathrm{Cu}$ site substitution with magnetic impurities influences polaron formation. Polarized $\mathrm{Cu} K$-edge EXAFS data were collected for the magnetic impurity-doped $\mathrm{La}_{1.85} \mathrm{Sr}_{0.15} \mathrm{Cu}_{1-x} \mathrm{M}_{x} \mathrm{O}_{4}$ (M $=\mathrm{Mn}, \mathrm{Ni}, \mathrm{Co}$ ) single-crystal samples. Temperature dependence of the in-plane $\mathrm{Cu}-\mathrm{O}$ DP is shown in Figures 8(a) $(\mathrm{M}=\mathrm{Ni}, \mathrm{Co})$ and $8(\mathrm{~b})(\mathrm{M}=\mathrm{Mn})$. Doping of impurities leads to different perturbations of the local lattice distortion (DP). Introducing a small amount of $\mathrm{Ni}$ and $\mathrm{Co}$ impurities 


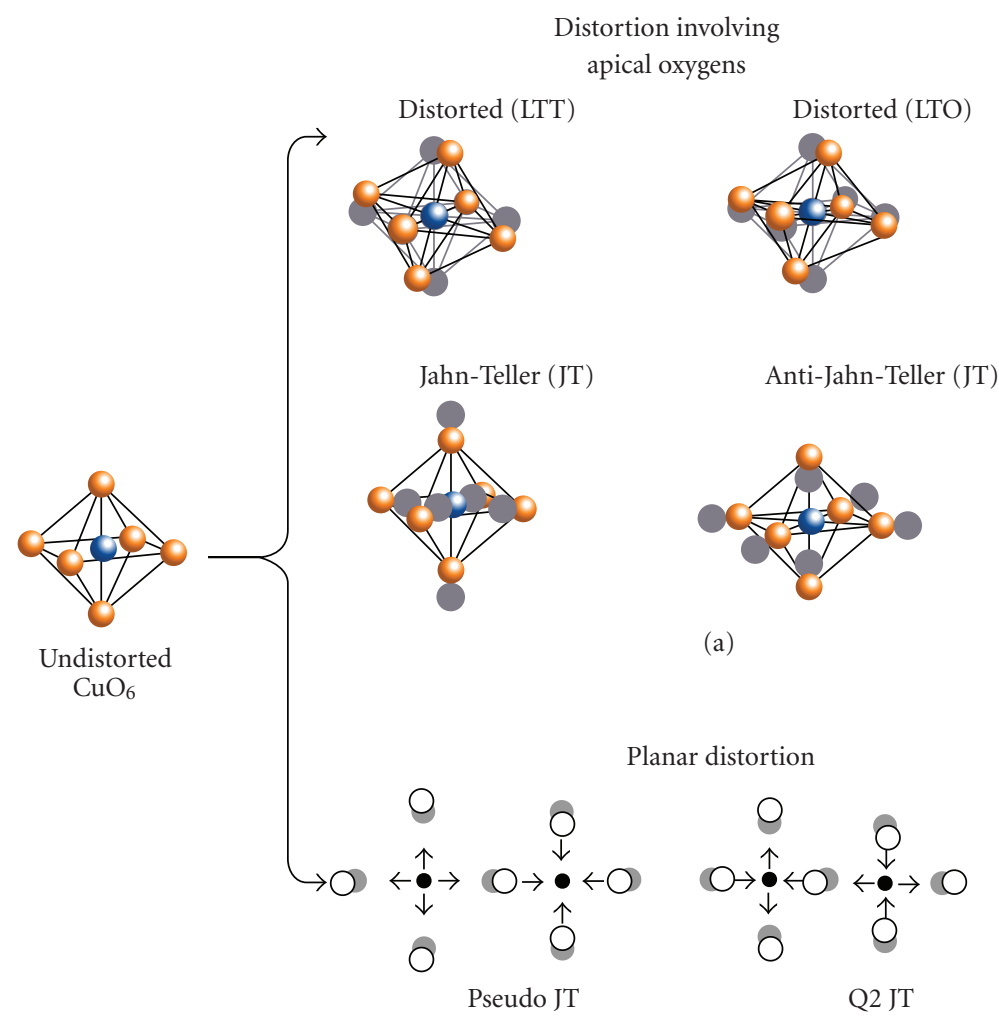

(b)

FIGURE 7: Schematic local lattice distortions. (a) Various single site deformation schemes involving apical oxygen displacement. (b) correlated deformation schemes with in-plane atomic displacements are schematically illustrated. In case of pseudo JT distortion model (left), oxygen atoms are distorted in a similar fashion with a breezing mode where all $\mathrm{Cu}-\mathrm{O}$ bonds for each $\mathrm{Cu}$ are either elongated or shortened. In the case of $\mathrm{Q}_{2}$ type JT distortion, half of $\mathrm{Cu}-\mathrm{O}$ bonds are either elongated or shortened.

at the $\mathrm{Cu}$ site sensitively depresses the unusual upturn of the $\mathrm{Cu}-\mathrm{O}$ DP. With $5 \%$ of $\mathrm{Ni}$ or Co substitution, the anomaly completely disappears with collapse of superconductivity. In contrast, substitution of $\mathrm{Mn}$ has less perturbation on lattice distortion for which superconductivity persists or the onset superconducting temperature is conserved. Magnetic impurities are thus detrimental to superconductivity in accordance with pair breaking theory. Such a magnetic scattering (pair breaking) has no indication of polaron formation but magnetic effect is weakened with the presence of polaron (Mn doping). The onset temperature of in-plane $\mathrm{Cu}-\mathrm{O} \mathrm{DP}$ was constant $(T=80 \mathrm{~K})$ for all Mn-doped LSCO samples. With increasing Mn doping, the magnitude of DP slightly decreases but the contribution of superconducting coherence persists in all samples up to $x=0.05$, indicating that $\mathrm{Mn}$ doping does not suppress polaron formation. In Niand Co-doped LSCO samples, in contrast, the magnitude of DP and superconducting phase coherence (superfluid density) decrease with increasing doping concentration. The preference of elongated in-plane M-O bonds (anti JTlike local environments) around an impurity atom may contribute to unperturbed antiferromagnetic correlation length [38].

3.5. Possible Models. The effect of superconducting coherence probed by DP (proportional to polaron density) precisely coincides with the inflection point of resistivity derivative $\partial \rho / \partial T$. This suggests that the onset of HTSC is induced as the density of metallic domains exceeds the critical value or the polaron density has a critical value for coherence. Let us consider a simple model (Figure 9) where the distance between polaronic domains $L$ and the superconducting coherence length $\zeta_{0}$ are key parameters. As doping proceeds, reduced Coulomb repulsion $(U)$ would promote integration into extended domains with a larger size. The average distance between extended domains $L$ decreases allowing tunnelling over insulating domains [15, 35]. When $L$ becomes greater than a critical value $(\zeta)$, quantum tunnelling is prohibited. Suppressed tunnelling under tensile strain decreases superfluid density, and hence the critical temperature. In contrast, polaron may also create perturbations in spin configuration so that spin vortices are formed that grows into a macroscopic supercurrent [17].

The $\mathrm{Mn}$ ions at $\mathrm{Cu}$ sites clearly favor polaron formation while the Ni or Co doping is strongly harmful to both polaron and superconductivity. This suggests that the dynamic nature of polaron is needed to conserve the superconducting transition. We found that the $\mathrm{Mn}$ impurities have elongated $\mathrm{M}-\mathrm{O}$ bond which could be favorable for distortions that cost elongation of bond length such as anti JT distortion [39]. Near the Mn dopants, more and more nonsuperconducting regions are formed and 


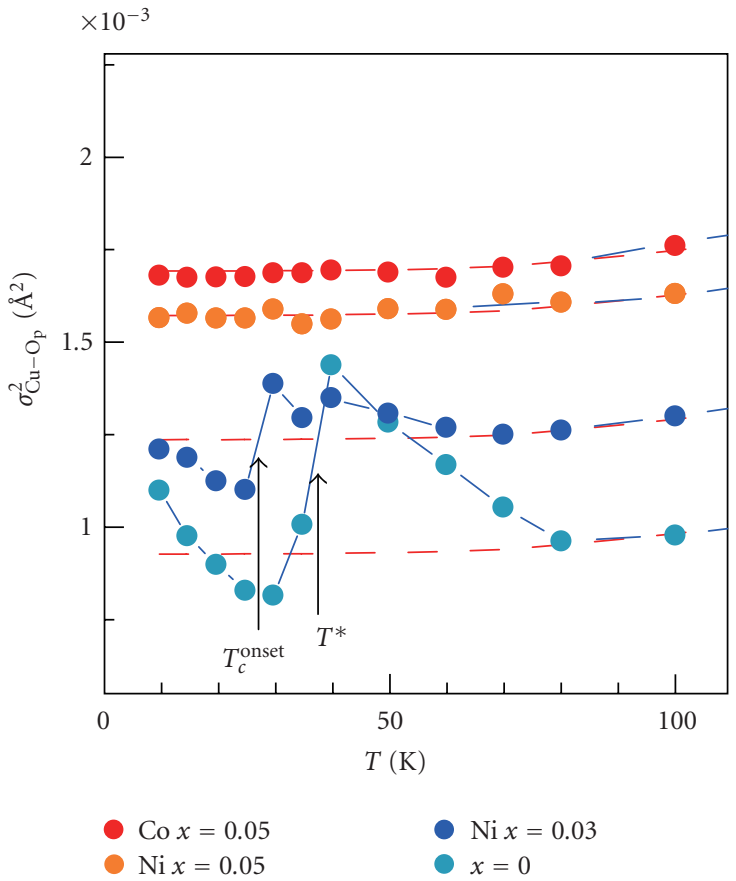

(a)

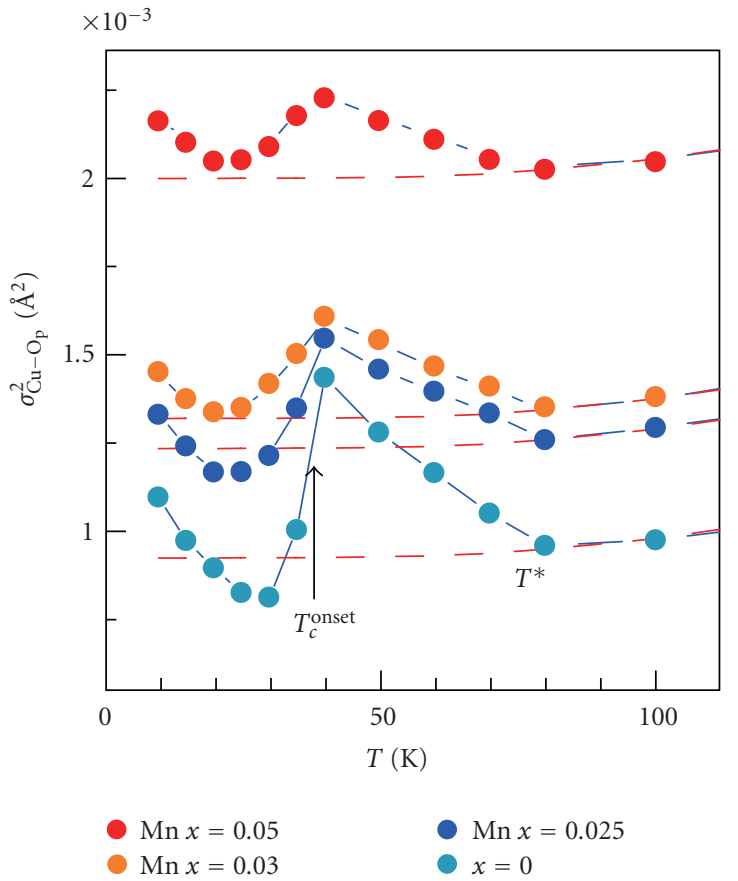

(b)

FIGURE 8: Mean-square relative displacement of the in-plane $\mathrm{Cu}-\mathrm{O}$ bond in magnetic impurity doped $\mathrm{M}_{\mathrm{L}} \mathrm{La}_{1-x} \mathrm{Sr}_{x} \mathrm{CuO}_{4}(x=0.15)$ as a function of temperature. (a) $\mathrm{M}=\mathrm{Co}, \mathrm{Ni}$ an (b) $\mathrm{M}=\mathrm{Mn}$, taken from [11].

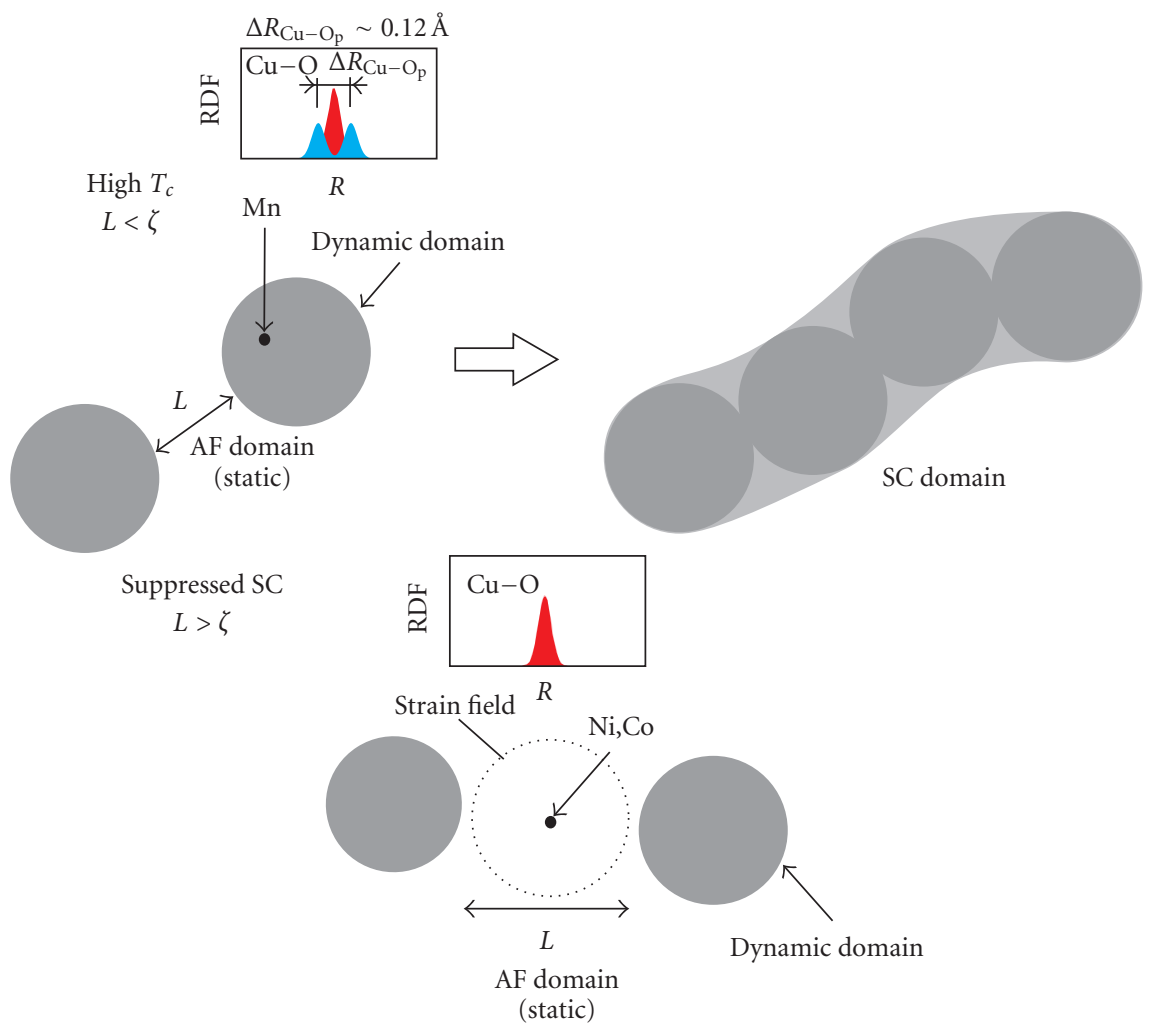

FIGURE 9: Schematic representation of quantum tunnelling in magnetic impurity doped $\mathrm{La}_{1-x} \mathrm{Sr}_{x} \mathrm{CuO}_{4}(x=0.15)$. 
superconducting domains are divided into small superconducting domains but within these domains polarons are conserved. As the distance between the superconducting domains becomes large, quantum tunneling or percolation is prohibited and the superconductivity eventually disappears.

Deeper knowledge on the location of doped hole and details of polaron, that is, formation, development and its role in electrical conduction is urgently needed.

\section{Conclusion}

The local lattice of $\mathrm{LaFeAsO}_{1-x} \mathrm{~F}_{x}$ superconductors was studied by X-ray absorption spectroscopy, EXAFS. We find an anomalous upturn of the mean-square relative displacement of the nearest neighbor ( $\mathrm{Fe}-\mathrm{As})$ bond below $T^{*}$ as electron carriers are introduced. The Fe-As local lattice fluctuation reveals the formation of two distinct bond lengths $R_{1}$ and $R_{2}$ where $\Delta R=R_{1}-R_{2} \sim 0.1 \AA$. The onset of lattice effects coincides with the opening of pseudogap. The results indicate that oxypnictide superconductors have lattice instability as a signature of polaron formation similar to that of HTSC cuprates. Refined EXAFS data for high-quality (thin films and bulk) $\mathrm{La}_{1-x} \mathrm{Sr}_{x} \mathrm{CuO}_{4}$ single crystals show that the relative oxygen displacement has characteristic features that is, inplane $\mathrm{Cu}-\mathrm{O}$ bond splitting indicating dynamical lattice distortion and a sharp drop due to coherence associated with the superconducting state. The observed magnitude of oxygen displacement is in good agreement with the charged cluster calculation on the $\mathrm{CuO}_{6}$ deformed pair [37]. Relevant lattice distortions for LSCO likely involve in-plane oxygen atoms as the apical oxygen atoms do not show anomalous displacements. We further find that substitution of $\mathrm{Mn}$ atom into a $\mathrm{Cu}$ site causes little perturbation of local lattice instability in contrast to $\mathrm{Ni}$ and Co substitutions which strongly suppress polaron formation and superconductivity. Suppression of superconductivity is related to the perturbation of local lattice, indicating that polaron formation drives the microscopic mechanism of superconductivity. Whether polaron works to perturb spin configurations leading to spin vortices growing into spin loop current [17] or it enhances strong coupling of spin, charge, and lattice [16] is an open question.

\section{Acknowledgments}

This work is collaborated with Y. Kamimura, H. Hosono, A. Tsukada, and M. Naito to whom the authors express their thanks for sample preparations. The authors also express their thanks to A. Bussmann-Holder, H. Koizumi, T. Kakeshita, H. Kamimura, J. Mustre de Leon, N. L. Saini, A. Bianconi, and A. Bishop for their continuous encouragement and fruitful discussions.

\section{References}

[1] J. G. Bednorz and K. A. Müller, "Possible high $T_{c}$ superconductivity in the Ba-La-Cu-O system," Zeitschrift für Physik B, vol. 64, no. 2, pp. 189-193, 1986.
[2] Y. Kamihara, T. Watanabe, M. Hirano, and H. Hosono, "Ironbased layered superconductor $\mathrm{La}\left[\mathrm{O}_{1-x} \mathrm{~F}_{x}\right] \mathrm{FeAs}(x=0.05-$ 0.12 ) with $T_{c}=26 \mathrm{~K}$," Journal of the American Chemical Society, vol. 130, no. 11, pp. 3296-3297, 2008.

[3] C. Fang, H. Yao, W.-F. Tsai, J. Hu, and S. A. Kivelson, "Theory of electron nematic order in LaFeAsO," Physical Review B, vol. 77, no. 22, Article ID 224509, 2008.

[4] P. W. Anderson, The Theory of Superconductivity in the Cuprates, Princeton University Press, Princeton, NJ, USA, 1997.

[5] W. Malaeb, T. Yoshida, T. Kataoka, et al., "Electronic structure and electron correlation in $\mathrm{LaFeAs}_{1-x} \mathrm{~F}_{x}$ and $\mathrm{LaFePO}_{1-x} \mathrm{~F}_{x}$," Journal of the Physical Society of Japan, vol. 77, no. 9, Article ID 093714, 2008.

[6] M. Hiraishi, R. Kadono, S. Takeshita, et al., "Full gap superconductivity in $\mathrm{Ba}_{0.6} \mathrm{~K}_{0.4} \mathrm{Fe}_{2} \mathrm{As}_{2}$ probed by muon spin rotation," Journal of the Physical Society of Japan, vol. 78, no. 2, Article ID 023710, 2009.

[7] A. S. Alexandrov, "Phase separation of electrons strongly coupled with phonons in cuprates and manganites," Journal of Superconductivity and Novel Magnetism, vol. 22, no. 2, pp. 95-101, 2009.

[8] N. Mannella, W. L. Yang, K. Tanaka, et al., "Polaron coherence condensation as the mechanism for colossal magnetoresistance in layered manganites," Physical Review B, vol. 76, no. 23, Article ID 233102, 2007.

[9] A. Lanzara, P. V. Bogdanov, X. J. Zhou, et al., "Evidence for ubiquitous strong electron-phonon coupling in hightemperature superconductors," Nature, vol. 412, no. 6846, pp. 510-514, 2001.

[10] C. J. Zhang, H. Oyanagi, Z. H. Sun, Y. Kamihara, and H. Hosono, "Low-temperature lattice structure anomaly in the $\mathrm{LaFeAs} \mathrm{O}_{0.93} \mathrm{~F}_{0.07}$ superconductor by X-ray absorption spectroscopy: evidence for a strong electron-phonon interaction," Physical Review B, vol. 78, no. 21, Article ID 214513, 2008.

[11] C. J. Zhang and H. Oyanagi, "Local lattice instability and superconductivity in $\mathrm{La}_{1.85} \mathrm{Sr}_{0.15} \mathrm{Cu}_{1-x} M_{x} \mathrm{O}_{4}(M=\mathrm{Mn}, \mathrm{Ni}$, and Co)," Physical Review B, vol. 79, no. 6, Article ID 064521, 2009.

[12] A. Bianconi, N. L. Saini, A. Lanzara, et al., "Determination of the local lattice distortions in the $\mathrm{CuO}_{2}$ Plane of $\mathrm{La}_{1.85} \mathrm{Sr}_{0.15} \mathrm{Cuo}_{4}$," Physical Review Letters, vol. 76, no. 18, pp. 3412-3415, 1996.

[13] N. L. Saini, A. Lanzara, H. Oyanagi, et al., "Local lattice instability and stripes in the $\mathrm{CuO}_{2}$ plane of the $\mathrm{La}_{1.85} \mathrm{Sr}_{0.15} \mathrm{CuO}_{4}$ system by polarized XANES and EXAFS," Physical Review B, vol. 55, no. 18, pp. 12759-12769, 1997.

[14] J. Mustre de Leon, S. D. Conradson, I. Batistić, and A. R. Bishop, "Evidence for an axial oxygen-centered lattice fluctuation associated with the superconducting transition in $\mathrm{YBa}_{2} \mathrm{Cu}_{3} \mathrm{O}_{7}$," Physical Review Letters, vol. 65, no. 13, pp. 16751678, 1990.

[15] D. Mihailovic, "Optical experimental evidence for a universal length scale for the dynamic charge inhomogeneity of cuprate superconductors," Physical Review Letters, vol. 94, no. 20, Article ID 207001, 4 pages, 2005.

[16] A. Bussmann-Holder and H. Keller, "Polaron formation as origin of unconventional isotope effects in cuprate superconductors," European Physical Journal B, vol. 44, no. 4, pp. 487490, 2005.

[17] H. Koizumi, "Loop current excitations in effectively half-filled mott insulators," Journal of the Physical Society of Japan, vol. 77, no. 3, Article ID 034712, 2008. 
[18] H. Oyanagi, C. Fonne, D. Gutknecht, et al., "Ge pixel array detector for high throughput X-ray spectroscopy," Nuclear Instruments and Methods in Physics Research A, vol. 513, no. 1-2, pp. 340-344, 2003.

[19] H. Oyanagi, A. Tsukada, M. Naito, and N. L. Saini, "Local structure of superconducting $(\mathrm{La}, \mathrm{Sr})_{2} \mathrm{CuO}_{4}$ under strain: Microscopic mechanism of strain-induced $T_{c}$ variation," Physical Review B, vol. 75, no. 2, Article ID 024511, 6 pages, 2007.

[20] H. Sato, M. Naito, and H. Yamamoto, "Superconducting thin films of $\mathrm{La}_{2} \mathrm{CuO}_{4+\delta}$ by oxygen doping using ozone," Physica $C$, vol. 280, no. 3, pp. 178-186, 1997.

[21] J.-P. Locquet, J. Perret, J. Fompeyrine, E. Mächler, J. W. Seo, and G. Van Tendeloo, "Doubling the critical temperature of $\mathrm{La}_{1.9} \mathrm{Sr}_{0.1} \mathrm{CuO}_{4}$ using epitaxial strain," Nature, vol. 394, no. 6692, pp. 453-456, 1998.

[22] I. Bozovic, G. Logvenov, I. Belca, B. Narimbetov, and I. Sveklo, "Epitaxial strain and superconductivity in $\mathrm{La}_{2-x} \mathrm{Sr}_{x} \mathrm{CuO}_{4}$ thin films," Physical Review Letters, vol. 89, no. 10, Article ID 107001, 2002.

[23] J. M. Tarascon, L. H. Greene, P. Barboux, et al., "3d-metal doping of the high-temperature superconducting perovskites La-Sr-Cu-O and Y-Ba-Cu-O," Physical Review B, vol. 36, no. 16, pp. 8393-8400, 1987.

[24] H. Oyanagi, A. Tsukada, M. Naito, et al., "Fluorescence Xray absorption spectroscopy using a Ge pixel array detector: application to high-temperature superconducting thin-film single crystals," Journal of Synchrotron Radiation, vol. 13, no. 4, pp. 314-320, 2006.

[25] J. J. Rehr, R. C. Albers, and S. I. Zabinsky, "High-order multiple-scattering calculations of X-ray-absorption fine structure," Physical Review Letters, vol. 69, no. 23, pp. $3397-$ 3400, 1992.

[26] C. de la Cruz, Q. Huang, J. W. Lynn, et al., "Magnetic order close to superconductivity in the iron-based layered $\mathrm{LaO}_{1-x} \mathrm{~F}_{x}$ FeAs systems," Nature, vol. 453, no. 7197, pp. 899$902,2008$.

[27] T. Nomura, S. W. Kim, Y. Kamihara, et al., "Crystallographic phase transition and high- $T_{c}$ superconductivity in LaFeAsO:F”, Superconductor Science and Technology, vol. 21, no. 12, Article ID 125028, 2008.

[28] J. Lee, K. Fujita, A. R. Schmidt, et al., "Spectroscopic fingerprint of phase-incoherent superconductivity in the underdoped $\mathrm{Bi}_{2} \mathrm{Sr}_{2} \mathrm{CaCu}_{2} \mathrm{O}_{8+\delta}$," Science, vol. 325, no. 5944, pp. 1099-1103, 2009.

[29] P. G. Radaelli, D. G. Hinks, A. W. Mitchell, et al., "Structural and superconducting properties of $\mathrm{La}_{2-x} \mathrm{Sr}_{x} \mathrm{CuO}_{4}$ as a function of Sr content," Physical Review B, vol. 49, no. 6, pp. 41634175, 1994.

[30] C. J. Zhang, H. Oyanagi, and C. H. Lee, "Single crystal growth and characterization of $\mathrm{La}_{2-x} \mathrm{Sr}_{x} \mathrm{CuO}_{4}$ with $\mathrm{Mn}$ doping," Physica C, vol. 468, no. 11-12, pp. 898-902, 2008.

[31] R. Khasanov, D. G. Eshchenko, H. Luetkens, et al., "Direct observation of the oxygen isotope effect on the inplane magnetic field penetration depth in optimally doped $\mathrm{YBa}_{2} \mathrm{Cu}_{3} \mathrm{O}_{7-\delta}$," Physical Review Letters, vol. 92, no. 5, Article ID 057602, 2004.

[32] R. J. McQueeney, Y. Petrov, T. Egami, M. Yethiraj, G. Shirane, and Y. Endoh, "Anomalous dispersion of LO phonons in $\mathrm{La}_{1.85} \mathrm{Sr}_{0.15} \mathrm{CuO}_{4}$ at low temperatures," Physical Review Letters, vol. 82, no. 3, pp. 628-631, 1999.

[33] L. Pintschovius and M. Braden, "Anomalous dispersion of LO phonons in $\mathrm{La}_{1.85} \mathrm{Sr}_{0.15} \mathrm{CuC}_{4}$," Physical Review B, vol. 60, no. 22, pp. R15039-R15042, 1999.
[34] T. Fukuda, J. Mizuki, K. Ikeuchi, K. Yamada, A. Q. R. Baron, and S. Tsutsui, "Doping dependence of softening in the bondstretching phonon mode of $\mathrm{La}_{2-x} \mathrm{Sr}_{x} \mathrm{CuO}_{4}(0 \leq x \leq 0.29)$," Physical Review B, vol. 71, no. 6, Article ID 060501, 2005.

[35] D. Mihailovic, V. V. Kabanov, and K. A. Müller, "The attainable superconducting $T_{c}$ in a model of phase coherence by percolating," Europhysics Letters, vol. 57, no. 2, pp. 254-259, 2002.

[36] J. C. Phillips, "Self-organized networks and lattice effects in high-temperature superconductors," Physical Review B, vol. 75, no. 21, Article ID 214503, 2007.

[37] S. Miyaki, K. Makoshi, and H. Koizumi, "Two-copper-atom units induce a pseudo Jahn-Teller polaron in hole-doped cuprate superconductors," Journal of the Physical Society of Japan, vol. 77, no. 3, Article ID 034702, 2008.

[38] H. Kamimura, H. Ushio, S. Matsuno, and T. Hamada, Theory of Copper Oxide Superconductors, Springer, Berlin, Germany, 2005.

[39] C. J. Zhang and H. Oyanagi, in preparation. 

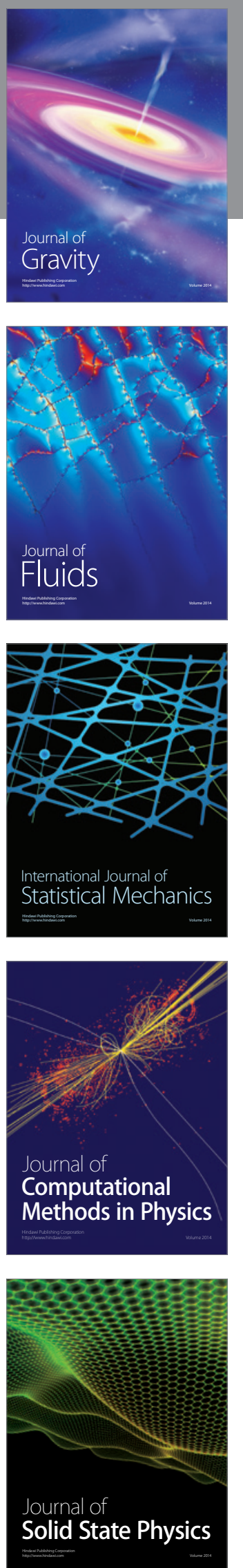

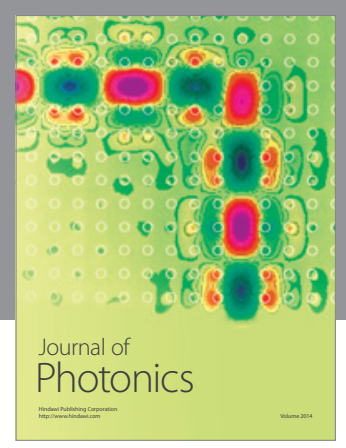

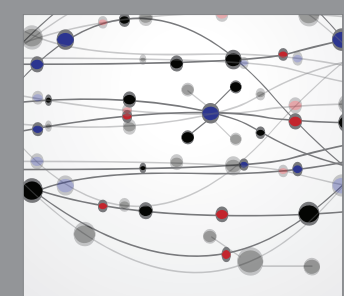

The Scientific World Journal
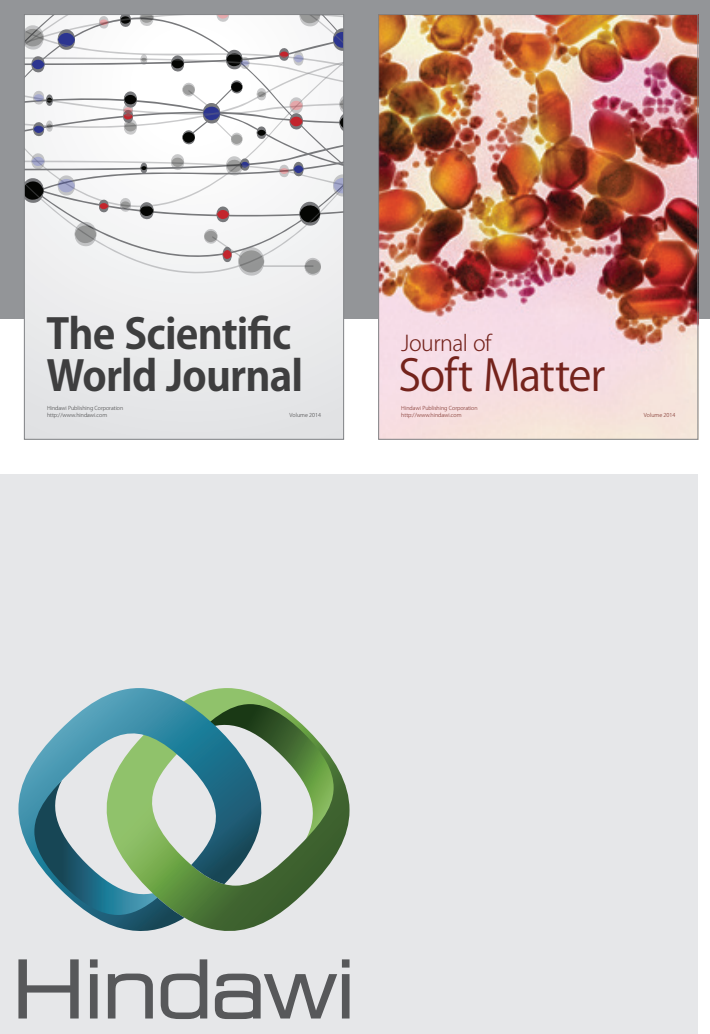

Submit your manuscripts at

http://www.hindawi.com
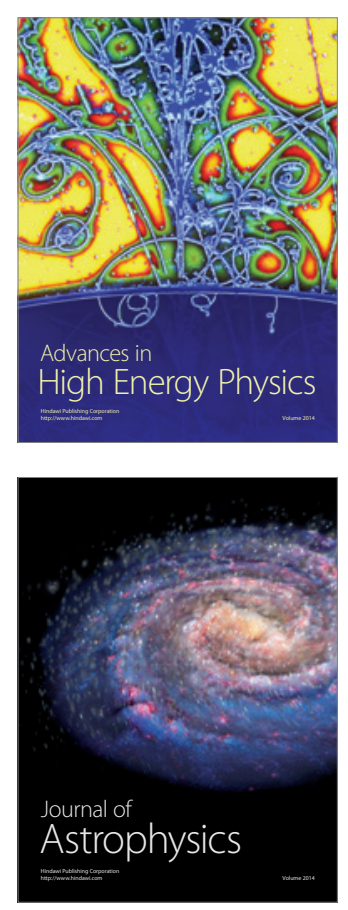
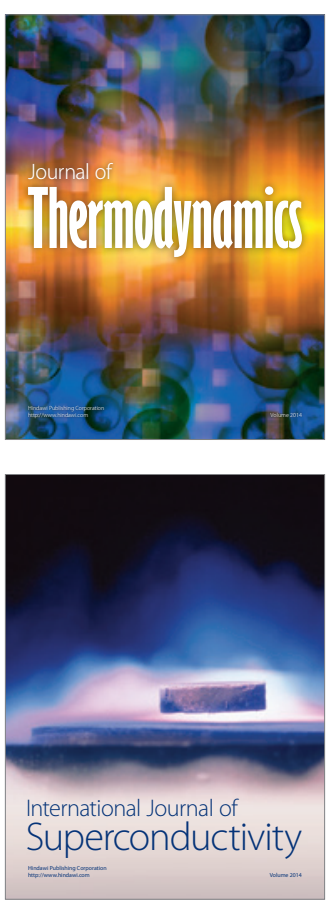
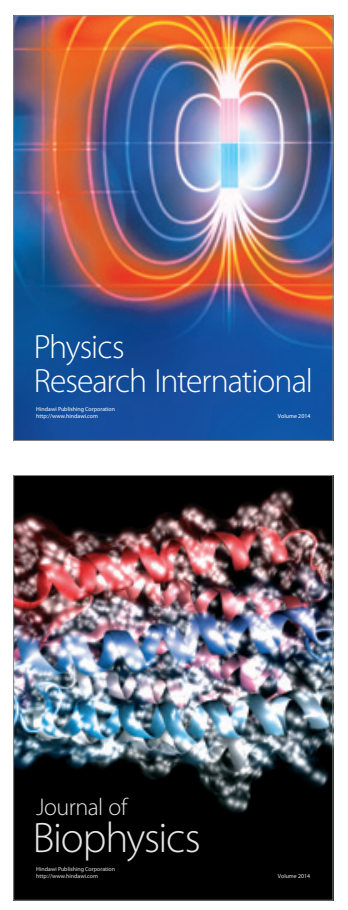
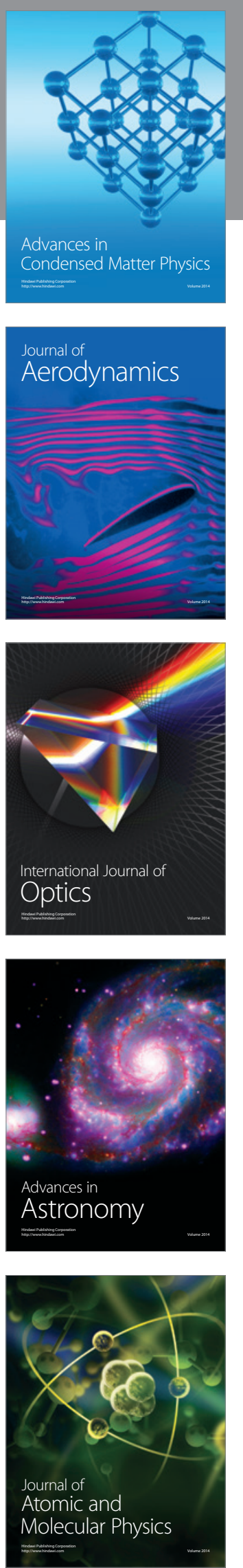